\title{
Controle Supervisório Hierárquico Baseado em Interface: Caso Mecatrime
}

\author{
Igor C. Calixto* Antonio E. C. da Cunha* \\ * Instituto Militar de Engenharia (IME), Programa de Pós-Graduação \\ em Engenharia Elétrica, RJ, (e-mail: igorcohen@hotmail.com, \\ carrilho@ime.eb.br).
}

\begin{abstract}
Industrial manufacturing systems are becoming more complex by inserting properties aligned with the precepts of industry 4.0. In this context, this article review the interfacebased hierarchical control (HISC) with a parallel architecture applied to the MECATRIME manufacturing system aiming, in this way, to impose a hierarchy that limits complexity and adds flexibility to the system.

Resumo: Sistemas de manufatura industrias estão, com o passar do tempo, se tornando mais complexos ao inserir propriedades alinhadas aos preceitos da indústria 4.0. Nesse contexto, esse artigo resgata o controle hierárquico baseado em interface (HISC) com uma arquitetura em paralelo por meio da sua aplicação ao sistema de manufatura MECATRIME objetivando, dessa forma, a imposição de uma hierarquia que limite a complexidade e adicione flexibilidade ao sistema.
\end{abstract}

Keywords: Discrete Event Systems; Supervisory Control; Hierarchical Interface-Based; Pallalel Architecture; Hierarchy.

Palavras-chaves: Sistemas a Eventos Discretos; Controle Supervisório; Hierarquias Baseadas em Interfaces; Arquitetura em Paralelo; Hierarquia.

\section{INTRODUÇÃO}

A nova tendência industrial, denominada indústria 4.0, possui inúmeras características que, ao associadas, contribuem para uma maior flexibilidade e descentralização do sistema fabril.

Nessa nova fase, sobressai-se o emprego dos sistemas ciberfísicos (CPS - Cyber-Physical Systems) que são estruturalmente compostos por uma parte física monitorada, controlada e integrada pela sua projeção cibernética, hospedada em um computador, e por um núcleo de comunicação, constituído por sensores, atuadores, redes e unidades de processamento.

Dentro das abordagens que lidam com os conceitos da indústria 4.0 destacam-se aquelas relacionadas aos Sistemas a Eventos Discretos (SEDs) (Cassandras and Lafoturne, 2008) em que distingui-se o emprego da Teoria de Controle Supervisório (TCS) (Ramadge and Wonham, 1989) de modo a sintetizar um agente de controle, denominado supervisor, que atue no sistema a ser controlado, permitindo a ocorrência de eventos que estão de acordo com um comportamento especificado.

Recentemente, o laboratório de mecatrônica sediado no Instituto Militar de Engenharia (IME) instalou o sistema de manufatura MECATRIME. Esse sistema é uma manufatura integrada por computador (CIM - Computer Integrated Manufacturing), que tem por objetivo demonstrar

^ O presente trabalho foi realizado com apoio da Coordenação de Aperfeiçoamento de Pessoal de Nível Superior - Brasil (CAPES) Código de Financiamento 001. as principais tecnologias da industria 4.0, contendo, dessa forma, estações de produção robotizadas que ilustram os principais processos encontrados na industria atual.

Ao investigar as camadas de decisões presentes no MECATRIME constatou-se a existência de uma hierarquia de comando, em que a estação de gerenciamento se comporta como um subsistema de nível superior coordenando subsistemas de níveis inferiores, correspondentes às outras estações.

Com o objetivo de adequar as características hierárquicas presentes no MECATRIME, foi realizada uma análise das principais técnicas associadas à TCS disponíveis na literatura que retratam essa particularidade. Entre elas, os trabalhos com perspectiva de síntese de baixo para cima (botton-up), ou seja, que buscam uma consistência por abstração construídas de um nível inferior até o superior, destacam-se (Zhong and Wonham, 1990), (Wong and Wonham, 1996), (da Cunha and Cury, 2008) e (Schmidt et al., 2004). Apesar dessas pesquisas apresentarem uma contribuição inegável, a hierarquia, com perspectiva de síntese de cima para baixo (top-down), ou seja, com uma concepção de abstração do nível superior para o inferior, é a que possui uma maior semelhança com as peculiaridades presentes no MECATRIME. Assim, este trabalho destina-se a resgata-la por meio do Controle Supervisório Hierárquico Baseado em Interface (Hierarchical Interfacebased Supervisory Control - HISC), proposto por Leduc et al. (2005a). Essa hierarquia configura o sistema em níveis bem definidos, com interfaces limitando as informações trocadas entre cada módulo e, consequentemente, o gerenciamento de cada nível sobre o outro. 
As principais arquiteturas que fundamentam-se em HISC são: o Caso em Série (SC - Serial Case) (Leduc et al., 2005a), que possui um único nível superior e inferior, o Caso em Paralelo (PC - Parallel Case) (Leduc et al., 2005b), com um único nível superior e múltiplos níveis inferiores e o Caso Multinível (ML- Multi-Level) (Hill et al., 2010), que dispõe de uma hierarquia com inúmeras ramificações e com apenas um nível superior.

De posse do embasamento bibliográfico, este trabalho apresenta o estudo de caso do sistema MECATRIME com sua implementação nos moldes do HISC e arquitetura em Paralelo (Leduc et al., 2005b) para apenas uma das ramificações do sistema. Ao final será realizada a comparação dos resultados obtidos com as técnicas usuais da TCS.

O artigo está organizado da seguinte forma: A seção 2 apresenta um conjunto de conceitos de SEDs e TCS importantes para entendimento do trabalho. A seção 3 apresenta as propriedades e definições do HISC e a arquitetura em paralelo proposta por Leduc et al. (2005b). A seção 4 exibe uma analise do sistema MECATRIME, de modo a explorar sua modelagem nos padrões de SED e a demonstração de uma hierarquia em paralelo com seus resultados. A seção 5 apresenta comentários finais e perspectivas de desenvolvimento futuro.

\section{PRELIMINARES}

Essa seção se baseia em (Cassandras and Lafoturne, 2008) e visa esclarecer acerca da terminologia e conceitos abordados em SEDs e TCS.

Os SEDs são sistemas dinâmicos com espaço de estado discreto e evolução regida pela ocorrência de eventos assíncronos e instantâneos. Existem vários formalismos para a modelagem desses sistemas, nesse trabalho utilizam-se as linguagens formais e os autômatos.

Nesse formalismo, alfabeto é um conjunto finito de símbolos correspondentes a eventos, denotado $\Sigma$. A justaposição ou concatenação de seus elementos é denominado palavra. O conjunto de todas as palavras de comprimento finito mais a palavra vazia, $\epsilon$, definidas sobre o alfabeto $\Sigma$, é denotado por $\Sigma^{*}$. Uma linguagem $L$ sobre o alfabeto $\Sigma$ é definida como sendo qualquer subconjunto de $\Sigma^{*}$. Ao analisarmos a palavra $s=p u$ verifica-se uma subdivisão dos símbolos que a compõem, em que p é denominado prefixo e $u$ sufixo de $s$. O fecho de prefixo de uma linguagem $L$ é o conjunto formado por todos os seus prefixos definido como $\bar{L} \triangleq\left\{s \in \Sigma^{*}:\left(\exists t \in \Sigma^{*}\right)[s t \in L]\right\} . \operatorname{Pwr}(\Sigma)$ denota o conjunto de partes de $\Sigma$, isto é, o conjunto que contem todos os seus subconjuntos, também denotado por $2^{\Sigma}$. O conjunto de eventos elegíveis em $L$ após $s$ é $\operatorname{Elig}_{L}(s) \triangleq$ $\{\sigma \in \Sigma \mid s \sigma \in L\}$, ou seja, o conjunto de eventos que podem suceder a $s$ em $L$. Quando não houver em $L$ nenhum evento elegível após a ocorrência de $s, \operatorname{Elig}_{L}(s)=\emptyset$.

Formalmente, um autômato é uma quíntupla representada por $G=\left(X, \Sigma, \delta, x_{0}, X_{m}\right)$, em que $X$ é o seu conjunto de estados, $\Sigma$ o alfabeto, $x_{0} \in X$ o estado inicial, $X_{m} \subseteq X$ o conjunto de estados marcados e $\delta: X \times \Sigma \rightarrow X$ a função de transição. Dado um símbolo $\sigma \in \Sigma$ e dois estados $x$ e $x^{\prime}$, então sempre que se escrever $x^{\prime}=\delta(x, \sigma)$, entende-se por um transição que sai de $x$, etiquetada por $\sigma$ e levando a $x^{\prime}$. Para denotar que uma transição é possível a partir de um certo estado $x$ por um evento $\sigma$ tem-se $\delta(x, \sigma)$ !.

A conexão entre autômatos e linguagens é facilmente identificada ao observar o sequenciamento de eventos a partir do estado inicial. A linguagem gerada pelo autômato $G$ é definida como $L(G) \triangleq\left\{s \in \Sigma^{*}: \delta\left(x_{0}, s\right)\right.$ ! $\}$, em que se usa a expansão da função de transição para palavras. A linguagem marcada pelo autômato $G$ é definida como $L_{m}(G)=\left\{s \in L: \delta\left(x_{0}, s\right) \in X_{m}\right\}$. Um autômato é acessível quando existem transições para todos os seus estados a partir do estado inicial.

Sejam dois alfabetos $\Sigma_{1}$ e $\Sigma_{2}$, sua união $\Sigma=\Sigma_{1} \cup \Sigma_{2}$ e as linguagens $L_{1} \subseteq \Sigma_{1}$ e $L_{2} \subseteq \Sigma_{2}$. A projeção de palavras $P_{i}$ pertencentes a um alfabeto $\Sigma$ em um subalfabeto $\Sigma_{i} \subseteq \Sigma$ é definida como $P_{i}: \Sigma^{*} \rightarrow \Sigma_{i}^{*}$, em que:

$$
\begin{aligned}
& P_{i}(\epsilon)=\epsilon, \\
& P_{i}(\sigma)=\left\{\begin{array}{lll}
\sigma, & \text { se } & \sigma \in \Sigma_{i} \\
\epsilon, & \text { se } & \sigma \in \Sigma / \Sigma_{i}
\end{array},\right. \\
& P_{i}(s \sigma)=P_{i}(s) P_{i}(\sigma) \text { para } s \in \Sigma^{*} \text { e } \sigma \in \Sigma \text {. }
\end{aligned}
$$

O produto síncrono entre as linguagens $L_{1}$ e $L_{2}$ é definido como $L_{1} \| L_{2}=P_{1}^{-1}\left(L_{1}\right) \cap P_{2}^{-1}\left(L_{2}\right)$ em que $P_{i}^{-1}: \Sigma_{i}{ }^{*} \rightarrow$ $\operatorname{Pwr}\left(\Sigma^{*}\right)$ é a imagem inversa da projeção.

A composição síncrona de dois subsistemas distintos de um SED, isto é, $G_{1}=\left(X_{1}, \Sigma_{1}, \delta_{1}, x_{01}, X_{1 m}\right)$ e $G_{2}=$ $\left(X_{2}, \Sigma_{2}, \delta_{2}, x_{02}, X_{2 m}\right)$, é denotado $G_{1} \| G_{2}$ e possui as seguintes propriedades para com as suas linguagens:

$$
\begin{gathered}
L\left(G_{1} \| G_{2}\right)=L\left(G_{1}\right) \| L\left(G_{2}\right) \text { e } \\
L_{m}\left(G_{1} \| G_{2}\right)=L_{m}\left(G_{1}\right) \| L_{m}\left(G_{2}\right) .
\end{gathered}
$$

Existem duas propriedades objetivadas ao se tratarem SEDs, o não bloqueio e a controlabilidade. Um autômato $G$ é dito ser não bloqueante, por definição, quando $\overline{L_{m}(G)}=$ $L(G)$, ou seja, todos os caminhos possíveis do autômato podem ser estendidos para alcançar estados marcados.

Para tratar o controle da planta de um sistema, definise o supervisor. Sua representação é o autômato $S=$ $\left(X, \Sigma_{S}, \delta, x_{0}, X_{m}\right)$ e seu comportamento ao supervisionar uma planta é tido como $G \| S$. Um supervisor $S$ para $G$ é dito ser não bloqueante se $S \| G$ for não bloqueante.

A partir do alfabeto formado pela união disjunta dos eventos não controláveis e controláveis, $\Sigma=\Sigma_{u} \cup \dot{\cup} \Sigma_{c}$, temse a definição formal de controlabilidade:

Definicão 1. Sejam as linguagens $L=\bar{L} \subseteq \Sigma^{*}$ e $K \subseteq \Sigma^{*}$ e o alfabeto $\Sigma=\Sigma_{u} \dot{\cup} \Sigma_{c}$. Desse modo, $K$ é Controlável e.r.a. $L$ e $\Sigma_{u}$ s.s.s. $(\forall s \in \bar{K} \cap L) \operatorname{Elig}_{L}(s) \cap \Sigma_{u} \subseteq \operatorname{Elig}_{\bar{K}}(s)$ ou $\bar{K} \Sigma_{u} \cap L \subseteq \bar{K}$.

Dado uma planta $G$, com conjunto de eventos $\Sigma=\Sigma_{u} \dot{\cup} \Sigma_{c}$, e uma linguagem $K \subseteq L_{m}(G)$, a condição necessária e suficiente para que exista um supervisor não bloqueante $S$ para $G$, tal que $L_{m}(S / G)=K$ é que $K$ seja controlável (e.r.a. $L(G)$ e $\left.\Sigma_{u}\right)$.

Se, ainda, $K$ não for controlável, é sempre possivel encontrar a suprema sublinguagem controlável de $K, \sup C(K)$, e.r.a. $L(G)$ e $\Sigma_{u}$ e, caso $\sup C(K)$ seja não vazio, existir um supervisor não bloqueante $S$ para $G$ tal que $L_{m}(S / G)=$ $\sup C(K)$. 


\section{CONTROLE SUPERVISÓRIO HIERÁRQUICO BASEADO EM INTERFACE}

O HISC é caracterizado por uma relação mestre e escravo, em que o subsistema de nível superior envia perguntas ou pedidos exclusivos para cada subsistema de nível inferior conectado a ele, a partir disso, esse subsistema executa o pedido e envia uma resposta, indicando sua conclusão. A interação feita entre um subsistema de nível superior e inferior é limitada por uma interface particular, de modo que uma pergunta deve ser respondida antes que uma nova seja feita. Essa seção se baseia nos estudos de (Leduc et al., 2005a) e (Leduc et al., 2005b).

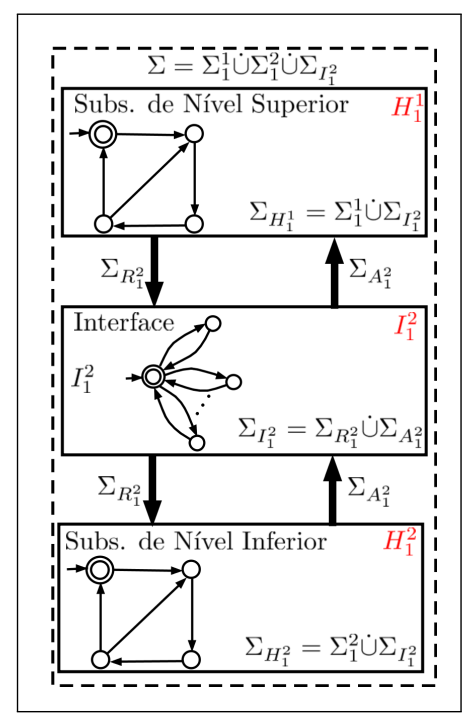

Figura 1. Diagrama Interface.

Tomando por base o caso com apenas um nível inferior e superior representado na figura 1 , divide-se o conjunto de eventos $\Sigma=\Sigma_{1}^{1} \dot{\cup} \Sigma_{1}^{2} \dot{\cup} \Sigma_{R_{1}^{2}} \dot{\cup} \Sigma_{A_{1}^{2}}$, em que $\Sigma_{1}^{1}$, com o índice acima representando o nível e o abaixo a ramificação da hierarquia, é o conjunto de eventos pertencente apenas ao subsistema de nível superior (High-Level), $\Sigma_{1}^{2}$ é o conjunto de eventos exclusivo do subsistema de nível inferior (LowLevel), $\Sigma_{R_{1}^{2}}$ é o conjunto de perguntas (Request) e $\Sigma_{A_{1}^{2}}$ é o conjunto de respostas (Answers). Os autômatos que representam o nível superior e inferior são, respectivamente, $H_{1}^{1}$ definido sobre $\Sigma_{1}^{1} \dot{\cup} \Sigma_{R_{1}^{2}} \dot{\cup} \Sigma_{A_{1}^{2}}$ e $H_{1}^{2}$ definido sobre $\Sigma_{1}^{2} \dot{\cup} \Sigma_{R_{1}^{2}} \dot{\cup} \Sigma_{A_{1}^{2}}{ }^{1} \mathrm{O}$ autômato $I_{1}^{2}$, que representa a interface, possui o conjunto de eventos $\Sigma_{I_{1}^{2}}=\Sigma_{R_{1}^{2}} \cup \Sigma_{A_{1}^{2}}$, tal que seus eventos são compartilhados apenas pelos subsistemas conectados por ela. Os eventos de $\Sigma_{R_{1}^{2}}$ correspondem as perguntas, ou pedidos, realizados pelo nível superior ao seu nível inferior; já os eventos $\Sigma_{A_{1}^{2}}$ são as respostas, ou reações, do nível inferior para as perguntas do seu nível superior. Esse trabalho considera todas as interfaces tratadas do tipo comando em par, definidas a seguir:

Definicão 2. O SED $I_{1}^{2}=\left(X, \Sigma_{R_{1}^{2}} \dot{\cup} \Sigma_{A_{1}^{2}}, \delta, x_{0}, X_{m}\right)$ é uma interface comando em par quando:

$$
\text { A) } L\left(I_{1}^{2}\right) \subseteq \overline{\left(\Sigma_{R_{1}^{2}} \Sigma_{A_{1}^{2}}\right)^{*}} \text {; e }
$$

\footnotetext{
1 Optou-se pela denominação dos termos High-Level e Low-Level em português por níveis superior e inferior, respectivamente.
}
B) $L_{m}\left(I_{1}^{2}\right)=\left(\Sigma_{R_{1}^{2}} \Sigma_{A_{1}^{2}}\right)^{*} \cap L\left(I_{1}^{2}\right)$.

Na definição 2, o item $A$ expressa que em $L\left(I_{1}^{2}\right)$ os eventos de pergunta se alternam com os de resposta, em sequências que iniciam-se por eventos de perguntas; o item $B$, que em $L_{m}\left(I_{1}^{2}\right)$ é composta de palavras vazias ou de palavras geradas em $I_{1}^{2}$ que finalizam por eventos de resposta. Dessa maneira, observa-se que $I_{1}^{2}$ deve ser composto por eventos disjuntos e que nunca haverá um estado marcado com eventos de resposta saindo dele.

\subsection{Caso em paralelo}

O Caso em Paralelo, figura 2, é esboçado por uma arquitetura com um único nível superior interagindo com $n \geq 1$ subsistemas de nível inferior em paralelo e independentes, por intermédio de suas respectivas interfaces.

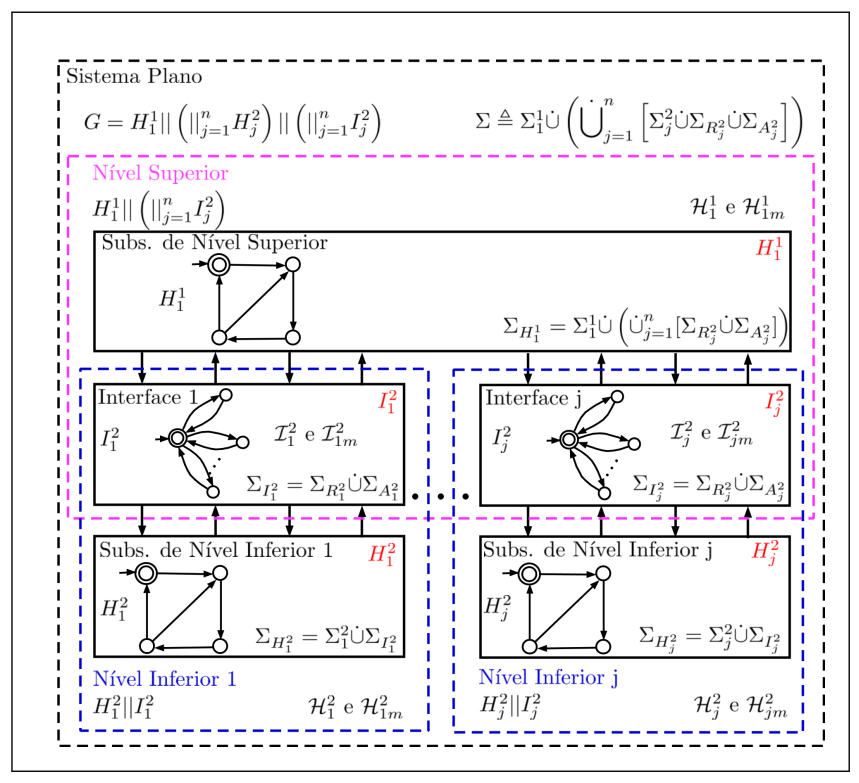

Figura 2. Arquitetura de um Sistema em Paralelo.

O alfabeto particionado do sistema plano, separado em subconjuntos disjuntos, é apresentado a seguir:

$$
\Sigma \triangleq \Sigma_{1}^{1} \dot{\cup}\left(\bigcup_{j=1}^{n}\left[\Sigma_{j}^{2} \dot{\cup} \Sigma_{R_{j}^{2}} \dot{\cup} \Sigma_{A_{j}^{2}}\right]\right) .
$$

O subsistema de nível superior representado pelo SED $H_{1}^{1}$ possui conjunto de eventos $\Sigma_{1}^{1} \dot{\cup}\left(\dot{\cup}_{j=1}^{n}\left[\Sigma_{R_{j}^{2}} \dot{\cup} \Sigma_{A_{j}^{2}}\right]\right)$. Seja $j \in\{1, \ldots, n\}$, cada subsistema de nível inferior $j$ é retratado, respectivamente, pelo SED $H_{j}^{2}$, definido sobre $\Sigma_{j}^{2} \dot{\cup} \Sigma_{R_{j}^{2}} \dot{\cup} \Sigma_{A_{j}^{2}}$ e por sua interface $I_{j}^{2}$, com o seguinte conjunto de eventos $\Sigma_{I_{j}^{2}}=\Sigma_{R_{j}^{2}} \cup \dot{U} \Sigma_{A_{j}^{2}}$.

O sistema plano para essa arquitetura é definido como $H_{1}^{1}\left\|\left(\|_{j=1}^{n} H_{j}^{2}\right)\right\|\left(\|\left.\right|_{j=1} ^{n} I_{j}^{2}\right)$. Além disso, ao dividirmos o sistema em planta e supervisor temos que o subsistema de nível superior é $H_{1}^{1}=G_{1}^{1} \| S_{1}^{1}$, cada subsistema de nível inferior $j$ é $H_{j}^{2}=G_{j}^{2} \| S_{j}^{2}$. A planta plana e o supervisor plano são apresentados a seguir:

$$
\begin{gathered}
G^{p} \triangleq G_{1}^{1} \|\left(\|_{j=1}^{n} G_{j}^{2}\right) \\
S \triangleq S_{1}^{1}\left\|\left(\|_{j=1}^{n} S_{j}^{2}\right)\right\|\left(\|\|_{j=1}^{n} I_{j}^{2}\right)
\end{gathered}
$$


Como forma de simplificar as notações é necessário introduzir os conjuntos de eventos, linguagens e projeções, apresentados nas tabelas 1, 2 e 3 .

Tabela 1. Conjuntos de Eventos e Projeções

\begin{tabular}{ccc}
\hline & Conjuntos de Eventos & Projeção \\
\hline Interface & $\Sigma_{I_{j}^{2}} \triangleq \Sigma_{R_{j}^{2}} \dot{\cup} \Sigma_{A_{j}^{2}}$ & $P_{I_{j}^{2}}: \Sigma^{*} \rightarrow \Sigma_{I_{j}^{2}}{ }^{*}$ \\
\hline Nível Superior & $\Sigma_{H_{1}^{1}} \triangleq \Sigma_{1}^{1} \dot{\cup}\left(\bigcup_{j=1}^{n} \Sigma_{I_{j}^{2}}\right)$ & $P_{H_{1}^{1}}: \Sigma^{*} \rightarrow \Sigma_{H_{1}^{1}}{ }^{*}$ \\
\hline Nível Inferior & $\Sigma_{H_{j}^{2}} \triangleq \Sigma_{j}^{2} \dot{\cup} \Sigma_{I_{j}^{2}}$ & $P_{H_{j}^{2}}: \Sigma^{*} \rightarrow \Sigma_{H_{j}^{2}}{ }^{*}$ \\
\hline
\end{tabular}

Tabela 2. Linguagens

\begin{tabular}{ccc}
\hline & Linguagem Gerada & Linguagem Marcada \\
\hline Interface & $\mathcal{I}_{j}^{2} \triangleq P_{I_{j}^{2}}^{-1}\left(L\left(I_{j}^{2}\right)\right)$ & $\mathcal{I}_{j m}^{2} \triangleq P_{I_{j}^{2}}^{-1}\left(L_{m}\left(I_{j}^{2}\right)\right)$ \\
\hline Nível Superior & $\mathcal{H}_{1}^{1} \triangleq P_{H_{1}^{1}}{ }^{-1}\left(L\left(H_{1}^{1}\right)\right)$ & $\mathcal{H}_{1 m}^{1} \triangleq P_{H_{1}^{1}}^{-1}\left(L_{m}\left(H_{1}^{1}\right)\right)$ \\
\hline Nível Inferior & $\mathcal{H}_{j}^{2} \triangleq P_{H_{j}^{2}}{ }^{-1}\left(L\left(H_{j}^{2}\right)\right)$ & $\mathcal{H}_{j m}^{2} \triangleq P_{H_{j}^{2}}{ }^{-1}\left(L_{m}\left(H_{j}^{2}\right)\right.$ \\
\hline
\end{tabular}

Tabela 3. Linguagens da Planta e Supervisor

\begin{tabular}{ccc}
\hline & Linguagem da Planta & Linguagem do Supervisor \\
\hline Nível Superior & $\mathcal{G}_{1}^{1} \triangleq P_{H_{1}^{1}}{ }^{-1}\left(L\left(G_{1}^{1}\right)\right)$ & $\mathcal{S}_{1}^{1} \triangleq P_{H_{1}^{1}}{ }^{-1}\left(L\left(S_{1}^{1}\right)\right)$ \\
\hline Nível Inferior & $\mathcal{G}_{j}^{2} \triangleq P_{H_{j}^{2}}{ }^{-1}\left(L\left(G_{j}^{2}\right)\right)$ & $\mathcal{S}_{j}^{2} \triangleq P_{H_{j}^{2}}{ }^{-1}\left(L\left(S_{j}^{2}\right)\right)$ \\
\hline
\end{tabular}

Observa-se que as linguagens $\mathcal{H}_{1}^{1}, \mathcal{H}_{j}^{2}$ e $\mathcal{I}_{j}^{2}$ são prefixo fechadas, dessa forma, a indicação do seu prefixo será desnecessárias nas expressões que serão apresentadas. Além disso, a razão para definir as linguagens usando projeções inversas nas tabelas 2 e 3 são essencialmente por questão de notação para as futuras expressões, em essência isso provoca que uma única palavra em $\Sigma^{*}$ pode ser visualizada por todos os níveis e interfaces sem a necessidade do uso de projeções.

Na definição a seguir são estabelecidas as propriedades necessárias para obter uma interface em paralelo consistente, isto é, que permita o funcionamento correto da hierarquia baseada em interface.

Definicão 3. O sistema com uma interface em paralelo constituído por $n \geq 1$ ramificações e composto pelos SEDs $H_{1}^{1}, H_{1}^{2}, \ldots, H_{j}^{2}, I_{1}^{2}, \ldots, I_{j}^{2}$, possui uma interface em paralelo consistente em relação ao alfabeto particionado do sistema plano (1), se as condições a seguir forem satisfeitas:

Propriedades de todos os níveis:

A) O alfabeto de $H_{1}^{1}$ é $\Sigma_{H_{1}^{1}}$ e o de cada $H_{j}^{2}$ é $\Sigma_{H_{j}^{2}}$; e

B) Cada interface $I_{j}^{2}$ é comando em par.

Propriedades do nível superior:

C) $\left(\forall s \in \mathcal{H}_{1}^{1} \cap\left(\bigcap_{k=1}^{n} \mathcal{I}_{k}^{2}\right)\right)$

$$
\operatorname{Elig}_{\mathcal{I}_{j}^{2}}(s) \cap \Sigma_{A_{j}^{2}} \subseteq \operatorname{Elig}_{\mathcal{H}_{1}^{1} \cap\left(\bigcap_{k \neq j} \mathcal{I}_{k}^{2}\right)}(s) .
$$

Propriedades do nível inferior:

D) $\left(\forall s \in \mathcal{H}_{j}^{2} \cap \mathcal{I}_{j}^{2}\right) \operatorname{Elig}_{\mathcal{I}_{j}^{2}}(s) \cap \Sigma_{R_{j}^{2}} \subseteq \operatorname{Elig}_{\mathcal{H}_{j}^{2}}(s)$;

E) $\left(\forall s \in \Sigma^{*} \Sigma_{R_{j}^{2}} \cap \mathcal{H}_{j}^{2} \cap \mathcal{I}_{j}^{2}\right)$

$$
\operatorname{Elig}_{\mathcal{H}_{j}^{2} \cap \mathcal{I}_{j}^{2}}\left(s \Sigma_{1}^{2^{*}}\right) \cap \Sigma_{A_{j}^{2}}=\operatorname{Elig}_{\mathcal{I}_{j}^{2}}(s) \cap \Sigma_{A_{j}^{2}} \text {, em que }
$$$$
\operatorname{Elig}_{\mathcal{H}_{j}^{2} \cap \mathcal{I}_{j}^{2}}\left(s \Sigma_{j}^{2^{*}}\right) \triangleq \cup_{l \in \Sigma_{j}^{2 *}} \operatorname{Elig}_{\mathcal{H}_{j}^{2} \cap \mathcal{I}_{j}^{2}}(s l) ; \mathrm{e}
$$

F) $\left(\forall s \in \mathcal{H}_{j}^{2} \cap \mathcal{I}_{j}^{2}\right) s \in \mathcal{I}_{j m}^{2} \Rightarrow\left(\exists l \in \Sigma_{j}^{2^{*}}\right) s l \in \mathcal{H}_{j m}^{2} \cap \mathcal{I}_{j m}^{2}$.

As propriedades serão elucidadas a seguir:
A) O nível superior e inferiores só podem compartilhar entre si os eventos de perguntas e respostas contidos nas interfaces;

B) Cada interface $I_{j}^{2}$ deve obedecer a definição de interface comando em par;

C) Os eventos de resposta elegíveis pela interface $I_{j}^{2}$ sempre são aceitos pelo nível superior sincronizado com as interfaces $I_{k}^{2}$, para $k \neq j$. Dessa forma, $\mathcal{H}_{1}^{1} \cap\left(\bigcap_{k \neq j} \mathcal{I}_{k}^{2}\right) \Sigma_{A_{j}^{2}} \cap \mathcal{I}_{j}^{2} \subseteq \mathcal{H}_{1}^{1} \cap\left(\bigcap_{k \neq j} \mathcal{I}_{k}^{2}\right)$, ou seja, o nível superior em conjunto com as interfaces $k$ sincronizadas a ele são controláveis em relação a interface $j$ e aos eventos de resposta $\Sigma_{A_{j}^{2}}$;

D) O nível inferior sempre aceita um evento de pergunta se elegível pela sua interface. Dessa maneira, $\mathcal{H}_{j}^{2} \Sigma_{R_{j}^{2}} \cap$ $\mathcal{I}_{j}^{2} \subseteq \mathcal{H}_{j}^{2}$, ou seja, o nível inferior é controlável em relação a interface $j$ associada a ele e aos eventos de pregunta $\Sigma_{R_{j}^{2}}$;

E) Imediatamente após a ocorrência de um evento de pergunta todas as respostas previstas na interface devem sempre poder ocorrer no nível inferior, isto é, antes que qualquer novo evento do nível inferior ocorra; e

F) Qualquer palavra marcada pela interface e aceita pelo nível inferior deve poder ser estendida a uma palavra marcada pelo nível inferior.

\subsection{Não Bloqueio por Nível em Paralelo}

O conjunto de condições indispensáveis que acarretarão no teorema final de não bloqueio para um sistema em paralelo será exposto a seguir.

Definicão 4. O sistema com uma interface em paralelo constituído por $n \geq 1$ ramificações e composto pelos SEDs $H_{1}^{1}, H_{1}^{2}, \ldots, H_{n}^{2}, I_{1}^{2}, \ldots, I_{n}^{2}$ é não bloqueante por nivel se:

A) Não Bloqueio para o Nível Superior: $\mathcal{H}_{1}^{1} \cap\left(\bigcap_{j=1}^{n} \mathcal{I}_{j}^{2}\right)=\overline{\mathcal{H}_{1 m}^{1} \cap\left(\bigcap_{j=1}^{n} \mathcal{I}_{j m}^{2}\right)}$.

B) Não Bloqueio para o Nível inferior: $\mathcal{H}_{j}^{2} \cap \mathcal{I}_{j}^{2}=\overline{\mathcal{H}_{j m}^{2} \cap \mathcal{I}_{j m}^{2}}$.

A definição 4 estabelece pelo item A que o nível superior sincronizado com todas interfaces possui caminhos saindo de todos os seus estados que alcançam um estado marcado e o item B indica o mesmo, porém para o nível inferior conectado a sua única interface.

Com base na definição 4, foi possível a criação de um teorema geral que condensa todas as informações sobre o não bloqueio de sistemas com HISC para o caso em paralelo, apresentado a seguir:

Teorema 5. Se o sistema com uma interface em paralelo constituído por $n \geq 1$ ramificações e composto pelos SEDs $H_{1}^{1}, H_{1}^{2}, \ldots, H_{n}^{2}, I_{1}^{2}, \ldots, I_{n}^{2}$ for não bloqueante por nível e possuir uma interface em paralelo consistente em relação ao alfabeto particionado do sistema plano, então:

$$
\begin{gathered}
L(G)=\overline{L_{m}(G)}, \text { em que: } \\
G=H_{1}^{1}\left\|\left(\|\|_{j=1}^{n} H_{j}^{2}\right)\right\|\left(\|\|_{j=1}^{n} I_{j}^{2}\right) .
\end{gathered}
$$

O teorema 5 apresenta que, para um sistema em paralelo com $n$ ramificações ser não bloqueante, basta verificar se o subsistema de nível superior e cada subsistema de nível 
inferior $j$ conectado ao de nível superior sincronizado com as outras interfaces possuam uma interface em paralelo consistente e sejam não bloqueantes por nível. Dessa forma, a constatação de que o sistema é globalmente não bloqueante em relação ao sistema plano é feita localmente em cada módulo da hierarquia.

\subsection{Controlabilidade por Nível em Paralelo}

As condições que resultarão no teorema geral da controlabilidade para sistemas em paralelo serão apresentadas a seguir.

Definicão 6. O sistema com uma interface em paralelo constituído por $n \geq 1$ ramificações e composto pelos SEDs $G_{1}^{1}, G_{1}^{2}, \ldots, G_{n}^{2}, S_{1}^{1}, S_{1}^{2}, \ldots, S_{n}^{2}, I_{1}^{2}, \ldots, I_{n}^{2}$ é controlável por nível em relação ao alfabeto particionado do sistema plano, se para todo $j \in\{1, \ldots, n\}$ as condições a seguir são satisfeitas:

A) O alfabeto de $G_{1}^{1}$ e $S_{1}^{1}$ é $\Sigma_{H_{1}^{1}}$, de cada $G_{j}^{2}$ e $S_{j}^{2}$ é $\Sigma_{H_{j}^{2}}$ e de $I_{j}^{2}$ é $\Sigma_{I_{j}^{2}}$;

B) $\left(\forall s \in \mathcal{G}_{j}^{2} \cap \mathcal{S}_{j}^{2} \cap \mathcal{I}_{j}^{2}\right) \operatorname{Elig}_{\mathcal{G}_{j}^{2}}(s) \cap \Sigma_{u} \subseteq \operatorname{Elig}_{\mathcal{S}_{j}^{2} \cap \mathcal{I}_{j}^{2}}(s)$;

C) $\left(\forall s \in \mathcal{G}_{1}^{1} \cap \mathcal{S}_{1}^{1} \cap\left(\bigcap_{j=1}^{n} \mathcal{I}_{j}^{2}\right)\right) \operatorname{Elig}_{\mathcal{G}_{1}^{1} \cap\left(\bigcap_{j=1}^{n} \mathcal{I}_{j}^{2}\right)}(s) \cap$ $\Sigma_{u} \subseteq \operatorname{Elig}_{\mathcal{S}_{1}^{1}}(s)$.

Na definição 6 é estabelecido que para a hierarquia do sistema seja controlável por nível em paralelo é necessário que:

A) Os níveis da hierarquia compartilham entre si apenas os eventos contidos na interface;

B) Cada planta $G_{j}^{2}$ seja controlável em relação ao supervisor $S_{j}^{2}$ em sincronia com a interface $I_{j}^{2}$, isto é, $\left(\mathcal{S}_{j}^{2} \cap \mathcal{I}_{j}^{2}\right) \Sigma_{u} \cap \mathcal{G}_{j}^{2} \subseteq\left(\mathcal{S}_{j}^{2} \cap \mathcal{I}_{j}^{2}\right) ; \mathrm{e}$

C) A planta $G_{1}^{1}$ sincronizada com as interfaces $I_{j}^{2}$, para $j=1, \ldots, n$, seja controlável em relação ao supervisor $S_{1}^{1}$, isto é, $\mathcal{S}_{1}^{1} \Sigma_{u} \cap\left(\mathcal{G}_{1}^{1} \cap\left(\bigcap_{j=1}^{n} \mathcal{I}_{j}^{2}\right)\right) \subseteq \mathcal{S}_{1}^{1}$.

Dessa maneira, pode-se concluir que a perspectiva de supervisão é distribuída de maneira que, para o nível superior, a interface vai funcionar como uma planta a ser controlada e, para o nível inferior, a interface vai funcionar como um supervisor.

De posse da definição anterior foi desenvolvido o teorema geral de controlabilidade para sistemas com HISC para o caso em paralelo, apresentado a seguir.

Teorema \%. Se o sistema com uma interface em paralelo constituído por $n \geq 1$ ramificações e composto pelas plantas $G_{1}^{1}, G_{1}^{2}, \ldots, G_{n}^{2}$, supervisores $S_{1}^{1}, S_{1}^{2}, \ldots, S_{n}^{2}$ e interfaces $I_{1}^{2}, \ldots, I_{n}^{2}$, for controlável por nível e possuir uma interface em paralelo consistente em relação ao alfabeto particionado do sistema plano, então:

$$
\left(\forall s \in L\left(G^{p}\right) \cap L(S)\right) \operatorname{Elig}_{L\left(G^{p}\right)}(s) \cap \Sigma_{u} \subseteq \operatorname{Elig}_{L(S)}(s) .
$$

O teorema 7 apresenta que, se o sistema for controlável por nível e possuir uma interface em paralelo consistente em relação ao alfabeto particionado do sistema plano, então a planta plana (2) é controlável em relação ao supervisor plano(3), isto é, $L(S) \Sigma_{u} \cap L\left(G^{p}\right) \subseteq L(S)$. Dessa forma, a constatação de que o sistema como um todo é controlável em relação a planta plana é feita localmente em cada módulo da hierarquia.

\section{ESTUDO DE CASO E DISCUSSÃO}

Essa seção apresenta o estudo de caso do sistema de manufatura integrada por computador MECATRIME nos moldes da teoria exposta anteriormente.

O sistema MECATRIME é composto por nove estações integradas fisicamente por uma esteira, de modo que a sincronia desses componentes permite a fabricação de peças e conjuntos mecânicos de perfis variados. Sua estrutura é visualizada na figura 3 e subdividida nas seguintes estações: Armazém(E1), torno(E2), fresa(E3), solda(E4), metrologia(E5), montagem e inspeção visual(E6), gravação a laser(E7), controle da esteira(E8) e gerenciamento(E9).

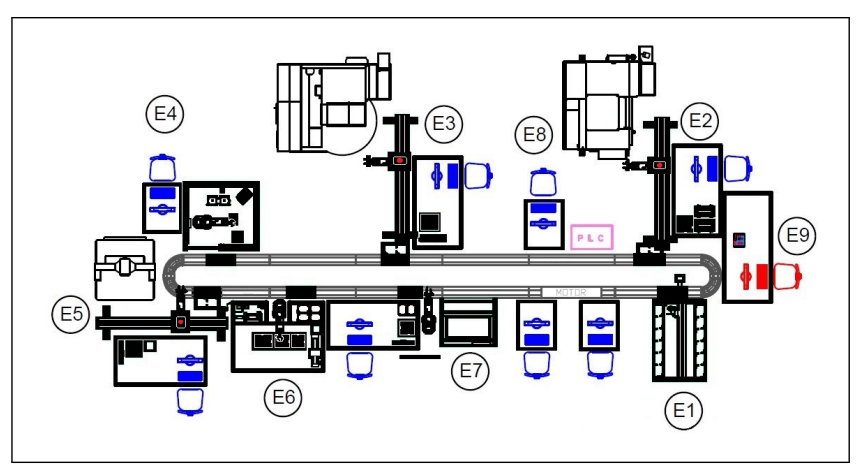

Figura 3. Sistema MECATRIME.

O foco do trabalho é fazer a análise isolada da interação da estação de gerenciamento com o torno, de modo que esse estudo seja replicável para as outras estações. Para isso, a produção local do torno será explorada, ou seja, os materiais necessários estarão a sua disposição sem o auxilio da estação de armazenamento e a esteira.

A estação do torno é composta por um robô manipulador de seis graus de liberdade, um trilho linear acoplado ao robô, de modo a aumentar sua área de atuação, um torno CNC (Computer Numeric Control), um armazém próprio para coleta de materiais e um rack para armazenamento da peça manufaturada.

Do ponto de vista físico, o sistema se comporta da seguinte maneira: o robô se move até a localização do armazém com o material a ser trabalhado, captura uma peça por meio de sua garra, o trilho linear se move até a localização do CNC, a porta da máquina se abre, o robô deposita a peça que é fixada por uma morsa, a porta se fecha, iniciase a manufatura, a porta se abre e o processo inverso é executado, porém finalizado com o deposito da peça no rack.

Do ponto de vista lógico, todo esse processo é controlado e integrado pela digitalização do sistema sediada nos softwares OPENCIM, SCORBASE e FS100, ou seja, uma perspectiva cibernética. A estação de gerenciamento por meio do software OPENCIM desempenha o papel de inicializar e acompanhar as rotinas do SCORBASE. Como consequência, o SCORBASE é um intermediário entre o OPENCIM e o FS100, o qual só executa seus comandos quando solicitado pelo SCORBASE.

Nesse trabalho a perspectiva cibernética será a escolhida, por apresentar uma similaridade com as técnicas exploradas. Desse modo, o SCORBASE possui um total de oito 
rotinas abordadas (GET023, PUT022, GET022, PUT025, PUTRACK, PLATHELO, PLATHEUN e CLLATHEDOOR) executadas sequencialmente a partir da solicitação de inicialização feita pela estação de gerenciamento e o FS100 possui nove outras (JOBGT023, JOBPT022, JOBGET022, PTGENER, LODOOR, OGRIP, CGRIP, LOCHUCK e LCCHUCK) a serem realizadas sempre que houver um pedido.

Inicialmente, a modelagem dessa estrutura lógica nos parâmetros dos sistemas a eventos discretos é feita por intermédio da análise isolada de cada rotina, como ilustrado na figura 4. Dessa forma, cada linha de código é interpretada como eventos que indicam verificação de sensores, comandos para os componentes no torno, conexão entre os softwares e definição de variáveis que por vezes foram refinados de modo a lidar com possíveis travamentos na sincronização. Esses eventos, por vezes são simplificados em apenas um, por representarem um único processo expandido em vários, como exemplo, na rotina GET023, figura 4, percebe-se a ocorrência de varias declarações para indicar a posição de um robô, dessa maneira elas foram simplificadas a um único evento correspondente a definição global dessas variáveis.

\begin{tabular}{|l|}
\hline \\
Set Subroutine GET023 \\
Print to Screen: GET FROM Gravity Feeder \\
Call Subroutine SCRIPT.GET_FROM_GFDR1 \\
Print to Screen: P1,P2, PB1: 'SCRIPT.P1', 'SCRIPT.P2', 'SCRIPT.PB1' \\
Print to Screen: P3,P4, P5: 'SCRIPT.P3', 'SCRIPT.P4', 'SCRIPT.P5' \\
Copy FS100 Position SCRIPT.P1 to Position 1001 \\
Copy FS100 Position SCRIPT.P2 to Position 1002 \\
Copy FS100 Position SCRIPT.P3 to Position 1003 \\
Copy FS100 Position SCRIPT.P4 to Position 1004 \\
Go to Position SCRIPT.PB1 Speed 50 (\%) \\
FS100 Start Job GT023 \\
Wait FS100_DELAY_TIME (10ths of seconds) \\
FS100 Job Wait 60 (seconds) \\
Send Message \$Start to MANAGER ID=TASK_ID \\
Return from Subroutine
\end{tabular}

Figura 4. Rotina GET023.

A rotina então é transformada em um autômato, figura 5, em que sua evolução estabelece a sequência de funcionamento correta de determinada função. Com isso, observase que o autômato obtido se comporta na maior parte das vezes de maneira sistemática, ou seja, executando os comandos ordenadamente restringindo de certa forma o sistema. Como consequência, essas rotinas são interpretadas como especificações de funcionamento do sistema, salvo apenas pelas rotinas PLATHELO e PLATHEUN que apresentam características que as configuram como plantas.

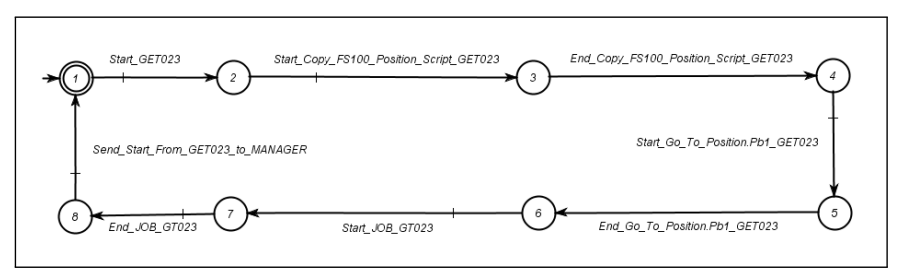

Figura 5. SED GET023.

Em virtude da interpretação das rotinas como especificações foi necessário a modelagem de plantas que agregassem maior permissividade ao sistema e que indicassem, dessa forma, o comportamento correto dos sensores, dos atuadores (robô, garra, porta do CNC e Morsa do CNC) e da inicialização do sistema. De posse dessa modelagem a tabela 4 apresenta o resultado inicial com o conjunto de autômatos que caracterizam a estação do torno.

Tabela 4. Plantas e Especificações

\begin{tabular}{cccc}
\hline Plantas & Estados/transi. & Especi. & Estados/transi. \\
\hline POSITION & $(9,16)$ & GET023 & $(8,8)$ \\
\hline ScorH11 & $(8,12)$ & JOBGT023 & $(14,14)$ \\
\hline Fs100H11 & $(5,8)$ & PUT022 & $(17,17)$ \\
\hline MOVH11 & $(5,8)$ & JOBPT022 & $(16,16)$ \\
\hline GrvtH11 & $(2,2)$ & GET022 & $(12,12)$ \\
\hline PLATHELO & $(3,3)$ & JOBGT022 & $(18,18)$ \\
\hline PLATHEUN & $(3,3)$ & PUT025 & $(4,4)$ \\
\hline VerLatheLoad & $(3,4)$ & PUTRACK & $(9,9)$ \\
\hline DoorH21 & $(3,5)$ & PTGENER & $(8,8)$ \\
\hline ScorDoorH21 & $(4,6)$ & ORDEM & $(10,10)$ \\
\hline VerLDClose & $(2,2)$ & CLLATHEDOOR & $(9,10)$ \\
\hline VerLDOpen & $(2,2)$ & LODOOR & $(8,11)$ \\
\hline VerCLDOpen & $(2,2)$ & OGRIP & $(3,9)$ \\
\hline VerCLDClose & $(2,2)$ & CGRIP & $(3,5)$ \\
\hline Fs100GripH22 & $(7,12)$ & LOCHUCK & $(8,11)$ \\
\hline GripH22 & $(3,6)$ & LCCHUCK & $(8,9)$ \\
\hline Fs100ChuckH23 & $(4,6)$ & & \\
\hline ChuckH23 & $(3,6)$ & & \\
\hline VerCchuckOff & $(2,2)$ & & \\
\hline VerCchuckOn & $(2,2)$ & & \\
\hline VerOchuckOff & $(2,2)$ & & \\
\hline VerOchuckOn & $(2,2)$ & & \\
\hline & & & \\
\hline
\end{tabular}

\subsection{Hierarquia Proposta}

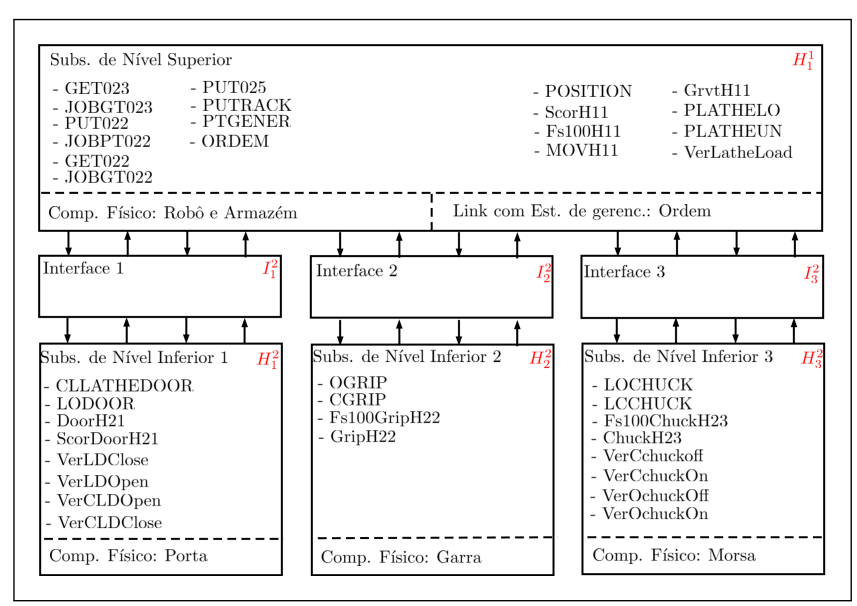

Figura 6. Hierarquia Proposta.

Com base na modelagem anterior, a hierarquia apresentada na figura 6 foi proposta de acordo com as técnicas apresentadas do HISC com uma arquitetura em paralelo. Para alcançar essa estrutura hierárquica foi realizada uma análise das rotinas e estabelecido o nível de comando que cada uma tem sobre a outra. A partir disso, foi verificada como deve ser a composição de cada módulo para que as propriedades apresentadas na seção anterior sejam respeitadas.

Para isso, verificou-se que o subsistema de nível superior deve conter todas as rotinas relacionadas ao Robô e armazém local do torno, de modo que a sua separação acarretaria em uma perda de consistência da interface, já que teríamos mais de um nível superior conectado a um inferior. O nível superior, necessita ainda de uma especificação que indique a ordem repassada pela estação de gerenciamento para o sequenciamento correto das rotinas. Esse subsistema possui, dessa forma, uma planta composta por POSITION || ScorH11 || Fs100H11 || MOVH11 || GrvtH11 || PLATHELO || PLATHEUN \| VerLatheLoad e uma especificação GET023 || JOBGT023 || PUT022 || 

JOBPT022 \| GET022 || JOBGT022 || PUT025 || PU-
TRACK | PTGENER \| ORDEM.

Além disso, os níveis inferiores, são correspondentes às rotinas que necessitam ser executadas em múltiplas ocasiões. Logo, o subsistema de nível inferior $H_{1}^{2}$ caracteriza a integração ciberfisíca dos softwares expostos com a porta do CNC, dessa maneira, sua planta apresenta a seguinte composição DoorH21 || ScorDoorH21 || VerLDClose || VerLDOpen || VerCLDOpen || VerCLDClose e especificação CLLATHEDOOR \| LODOOR.

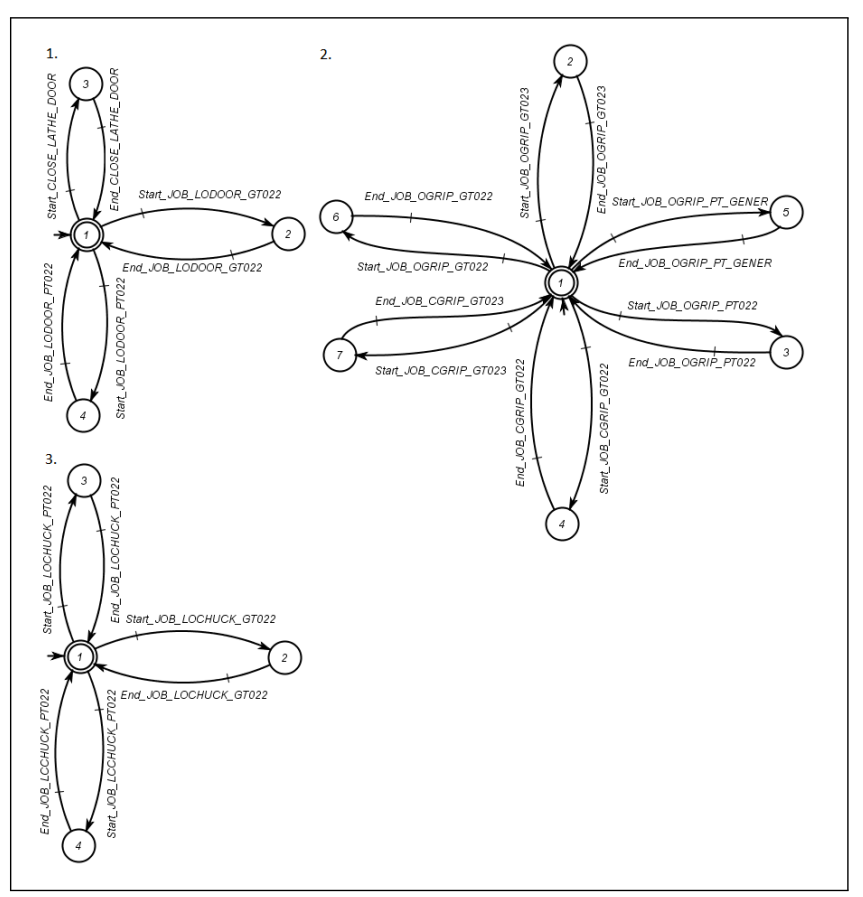

Figura 7. Interfaces $I_{1}^{2}, I_{2}^{2}$ e $I_{3}^{2}$.

O subsistema $H_{2}^{2}$ retrata a relação dos softwares com a garra acoplada ao robô dispondo de uma planta Fs100GripH22 || GripH22 e especificação OGRIP \| $C G R I P$. Da mesma forma, $H_{3}^{2}$ é associado com a morsa do CNC e possui uma planta constituída por Fs100ChuckH23 || ChuckH23 || VerCChuckOff || VerCChuckOn || VerOChuckOff || VerOChuckOn e especificação LOCHUCK \| LCCHUCK.

Após essa análise, foi realizada a verificação dos conjuntos de eventos respectivos a cada módulo e sua adequação com o alfabeto particionado do sistema plano (1). Em seguida, a definição dos eventos que correspondem ao conjunto de perguntas $\Sigma_{R_{j}^{2}}$ e de respostas $\Sigma_{A_{j}^{2}}$ foi feita, de modo que se obtiveram as interfaces apresentadas na figura 7 e que atendem a definição de interface comando em par. ${ }^{2}$

Com isso, a síntese dos supervisores é feita conforme as definições propostas na seção 3 , de modo que se obtenha a máxima linguagem controlável para HISC com arquitetura em paralelo. Para isso, realiza-se a síntese dos supervisores pela técnica monolítica da TCS em cada modulo da hierarquia e a partir do seu resultado é verificado se em algum requisito do HISC o sistema falha, caso não apresente nenhum problema o processo de síntese é finalizado. Por

2 Observa-se na figura 7 o refinamento dos eventos por meio de destinatários específicos para cada rotina solicitante no nível superior. outro lado, se o sistema não cumprir os pre-requisitos apresentados na seção anterior é realizado um processo cíclico de eliminação dos estados que falham em algum deles até que se alcance a linguagem característica do supervisor na condição da modelagem da hierarquia está correta. Dessa forma, os resultados são apresentados nas tabelas 5 e 6 .

Tabela 5. Plantas e Especificações

\begin{tabular}{ccc}
\hline & Planta & Especificação \\
\hline$H_{1}^{1}$ & $G_{1}^{1}(97200,1050840)$ & $E_{1}^{1}(96,96)$ \\
\hline$H_{1}^{2}$ & $G_{1}^{2}(192,1376)$ & $E_{1}^{2}(72,179)$ \\
\hline$H_{2}^{2}$ & $G_{2}^{2}(21,78)$ & $E_{2}^{2}(9,42)$ \\
\hline$H_{3}^{2}$ & $G_{3}^{2}(192,1440)$ & $E_{3}^{2}(64,160)$ \\
\hline
\end{tabular}

Tabela 6. Supervisor

\begin{tabular}{ccc}
\hline & $\mathrm{K}$ & Supervisor \\
\hline$H_{1}^{1}$ & $K_{1}^{1}=\left(G_{1}^{1}\left\|I_{1}^{2}\right\| I_{2}^{2} \| I_{3}^{2}\right) \| E_{1}^{1}(98,98)$ & $S_{1}^{1}(98,98)$ \\
\hline$H_{1}^{2}$ & $K_{1}^{2}=G_{1}^{2} \|\left(E_{1}^{2} \| I_{1}^{2}\right)(39,49)$ & $S_{1}^{2}(39,49)$ \\
\hline$H_{2}^{2}$ & $K_{2}^{2}=G_{2}^{2} \|\left(E_{2}^{2} \| I_{2}^{2}\right)(27,42)$ & $S_{2}^{2}(27,42)$ \\
\hline$H_{3}^{2}$ & $K_{3}^{2}=G_{3}^{2} \|\left(E_{3}^{2} \| I_{3}^{2}\right)(54,69)$ & $S_{3}^{2}(54,69)$ \\
\hline
\end{tabular}

\subsection{Verificação das Propriedades}

A verificação dos resultados foi realizada por uma ferramenta desenvolvida utilizando-se da biblioteca UltraDES (Martins et al., 2017). Para isso, adequou-se o algorítimo proposto por Leduc et al. (2005b) na implementação do teste.

Desse modo, constatou-se que a hierarquia proposta possui uma interface em paralelo consistente, ou seja, o sistema proposto respeita todas as propriedades expostas na seção 3 e está apto para o implemento da hierarquia.

\subsection{Discussão dos Resultados}

Ao analisar os supervisores obtidos foi legitimada a modelagem correta do sistema, de modo que sua constatação é ainda maior ao verificarmos que a composição das plantas com as interfaces e especificações, autômatos na coluna 2 da tabela 6 , são controláveis, ou seja, a especificação do nível superior e de cada nível inferior podem ser utilizadas como supervisores(Wonham and Cai, 2019).

Além disso, o alívio da explosão combinatorial de estados e o ganho em velocidade de processamento computacional foi constatado, tendo em vista que os valores de cada planta, autômatos na coluna 2 da tabela 5 , e supervisores, autômatos na coluna 3 da tabela 6 , são consideravelmente menores quando comparados com o caso monolítico, em que não foi possível determinar o tamanho do autômato da planta completa, por conta de seu tamanho e por falta de memória do computador, e o supervisor com 811 estados e 847 transições. Observou-se também que o supervisor monolítico é isomorfo ao supervisor plano do sistema com a arquitetura em paralelo do HISC o que significa que não houve perda de permissividade ao relacionar as duas técnicas.

\section{CONCLUSÃO}

O controle supervisório hierárquico baseado em interfaces em paralelo aplicado ao sistema de manufatura MECATRIME se comprovou uma solução para diminuir a complexidade computacional em relação as técnicas abordadas 
na teoria de controle supervisório, devido ao particionamento do sistema em múltiplos $(n \geq 1)$ subsistemas de nível inferior, proporcionando uma maior flexibilidade e independência dos níveis, como abordado anteriormente.

Um único limitador desses ganhos é o tamanho do nível superior por conta do produto síncrono entre o subsistema de nível superior e as interfaces de cada nível inferior $j$, podendo, dessa forma, crescer exponencialmente no número de componentes.

Apesar da arquitetura explorada ser em paralelo, o foco maior desse trabalho é resgatar essa técnica visando expansões futuras em toda a aplicabilidade do HISC. Desse modo, trabalhos seguintes terão como objetivo a implementação do sistema MECATRIME, como um todo, nos moldes de uma arquitetura em multinível e a procura por novas propriedades e arquiteturas viáveis por meio da expansão das abordagens tratadas no HISC, como: diminuição da complexidade da síntese; validação e melhora da lógica de controle existente; otimização do tempo de produção por exploração de estruturas hierárquicas; desenvolvimento de um sistema supervisório; interfaces que agreguem mais características ao sistema, ou uma nova arquitetura que englobe os conceitos presentes na indústria 4.0 .

\section{REFERÊNCIAS}

Cassandras, C.G. and Lafoturne, S. (2008). Introducion to Discrete Event Systems. Springer.

da Cunha, A.E.C. and Cury, J. (2008). Hierarchical supervisory control based on discrete event systems with flexible marking. IEEE Transactions on Automatic Control, 52, 2242 - 2253. doi:10.1109/TAC.2007.910653.

Hill, R., Cury, J., de Queiroz, M., Tilbury, D., and Lafortune, S. (2010). Multi-level hierarchical interface-based supervisory control. Automatica, 46(7), 1152-1164.

Leduc, R., Brandin, B., Lawford, M., and Wonham, W.M. (2005a). Hierarchical interface-based supervisory control, part i: Serial case. IEEE Transactions on Automatic Control, 50(9), 1322-1335.

Leduc, R., Brandin, B., Lawford, M., and Wonham, W.M. (2005b). Hierarchical interface-based supervisory control, part ii: Parallel case. IEEE Transactions on Automatic Control, 50(9), 1336-1348.

Martins, L.R.R., Dias, N.F.A., Alvesy, L.V.R., and Penaz, P.N. (2017). Ultrades 2.1 - uma biblioteca para modelagem, analise e controle de sistemas a eventos discretos. In XIII Simpósio Brasileiro de Automação Inteligente.

Ramadge, P.J.G. and Wonham, W.M. (1989). The control of discrete event systems. In Proceendings of the IEEE, volume 77, 81-98.

Schmidt, K., Reger, J., and Moor, T. (2004). Hierarchical control of structural decentralized des. In IFAC Proceedings Volumes, volume 37, 279-284.

Wong, K.C. and Wonham, W. (1996). Hierarchical control of discrete-event systems. Discrete Event Dynamic Systems: Theory and Applications, 6, 241-273. doi: 10.1007/BF01797154.

Wonham, W.M. and Cai, K. (2019). Supervisory Control of Discrete-Event Systems. Springer International $\mathrm{Pu}-$ blishing.

Zhong, H. and Wonham, W.M. (1990). On the consistency of hierarchical supervision in discrete-event systems.
IEEE Transactions on Automatic Control, 35(10), 11251134 . 